\title{
High frequency spikes in long period blood cell oscillations
}

\author{
Caroline Colijn • A. C. Fowler • \\ Michael C. Mackey
}

Received: 24 August 2005 / Revised: 5 May 2006 /

Published online: 2 August 2006

(C) Springer-Verlag 2006

\begin{abstract}
Several hematological diseases are characterised by oscillations of various blood cell populations. Two of these are a variant of chronic myelogenous leukemia (CML) and cyclical neutropenia (CN). These oscillations typically have long periods ranging from 20 to 60 days, despite the fact that the stem cell cycling time is thought to be of the order of 2-3 days. Clinical data from humans and laboratory data from the grey collie animal model of $\mathrm{CN}$ is suggestive of the idea that these long period oscillations may also contain higher frequency spiky oscillations. We show how such oscillations can be understood in the context of slow periodic stem cell oscillations, by analysing a two component differential-delay equation model of stem cell and neutrophil populations.
\end{abstract}

Keywords Stem cells · Chronic myelogenous leukemia - Cyclical neutropenia · Blood cell oscillations · Differential delay equations

For Karl Hadeler, on his 70th birthday, leader, teacher, colleague and friend.

C. Colijn $(\bowtie)$

Department of Mathematics and Centre for Nonlinear Dynamics, McGill University, 3655 Promenade Sir William Osler, Montreal, QC, Canada H3G 1Y6

e-mail: ccolijn@gmail.com

\section{A. C. Fowler}

Mathematical Institute,

24-29 St. Giles', Oxford OX1 3LB, UK

M. C. Mackey

Departments of Physiology, Physics and Mathematics, Centre for Nonlinear Dynamics, McGill University, 3655 Promenade Sir William Osler, Montreal, QC, Canada H3G 1Y6 


\section{Introduction}

There are several hematological diseases in which there are significant oscillations in blood cell numbers [11]. While some aspects of the oscillations are understood by biologists and modellers to result from destabilizations of the delayed feedback regulations that control cell numbers, the precise nature and origin of some of the oscillations remains unclear. This is in part due to the fact that the hematopoietic stem cells, from which all blood cells originate, are not easily accessible (nor even easy to define), and so the dynamics of the stem cells remain 'hidden'. Knowledge of the effects of changes in the dynamics of the stem cell compartment can be monitored indirectly through counts of the numbers of circulating white, red and platelet cells.

Colijn and Mackey [4] presented a model of blood cell production that contains the hematopoietic stem cells and three cell lines: the white, red and platelet cells, together with regulating negative feedback loops. In Colijn and Mackey $[4,5]$, this model was fitted to observed clinical data for oscillating white blood cell, reticulocyte and platelet numbers. These data came from cases of cyclical neutropenia $(\mathrm{CN})$ and periodic chronic myelogenous leukemia (PCML).

In the course of these simulations and comparisons, it appeared that there were high frequency oscillations riding on top of a dominant, lower-frequency oscillation in both the simulations and also the laboratory and clinical data. Because the nature of oscillations in the circulating cell numbers has implications for several diseases as well as for the dynamics of the less observable stem cells, the issue arises as to whether the observed high frequency spikes are manifestations of noise, or are genuine artifacts of the dynamics of the system. In this paper we examine the thesis that they are indicators of dynamical behavior.

The outline of this paper is as follows. First, in Sect. 2 we examine the available data to describe the nature of these high-frequency oscillations. Section 3 briefly presents the model of Colijn and Mackey [4,5]. Then in Sect. 4 we use this model to understand the theoretical basis for these oscillations. The paper concludes with a short discussion in Sect. 5.

\section{Spiky data in blood cell oscillations}

We have data available from two different diseases: Cyclical neutropenia in both the grey collie animal model and in humans, and periodic chronic myelogenous leukemia in humans. These data are in general oscillatory, and often show the high frequency osillations we will examine in this paper. In this section we present and discuss these data.

We have $\mathrm{CN}$ data from a group of grey collie dogs and from 27 neutropenic human patients. The human patients, who were diagnosed with severe idiopathic neutropenia, cyclical neutropenia or congenital neutropenia, were participants in a clinical trial of granulocyte-colony stimulating factor (G-CSF) [6]. Their data have been previously analysed [12]. For these patients, the sampling times were too sparse to show the high-frequency oscillations investigated here. We 
therefore focus on the dog data for cyclical neutropenia, and on human data for periodic chronic myelogenous leukemia.

\subsection{Cyclical neutropenia in the grey collie}

Our dog data were supplied by Prof. David C. Dale (University of Washington School of Medicine, Seattle). The dogs (grey collies) are all neutropenic, and were kept in temperature-controlled environments. Blood specimens were drawn daily. The dogs underwent a variety of treatments, and at times were not treated at all; thus we have data for treated dogs as well as untreated, neutropenic, dogs. Some of these data have been previously analysed by Haurie et al. [13].

However, there are 14 places in the dog data where we observe what appears to be a high-frequency oscillation in the neutrophils (white blood cells), on top of the dominant, slower oscillation typical of cyclical neutropenia. These points occurred primarily when the dogs were being treated with G-CSF. GCSF is a naturally occurring cytokine known to increase neutrophil numbers through an inhibition of apoptosis [2,17,20,24,25], but whose overall effect on the hematological system is not fully characterised.

In Fig. 1, we have plotted the neutrophil counts in grey collies where a visual inspection has identified high-frequency oscillations imposed on top of the 15-20 day period oscillations characteristic of $\mathrm{CN}$ in these animals [13]. In Fig. 2 we show the leukocyte counts for patients with PCML (see Sect. 2.2). The behavior we are interested in here is typified by the plot labelled 'dog 113 3', which clearly shows high frequency oscillations at the peak of the neutrophil count but not at the nadir.

The observation that the data may show higher-frequency variations, primarily near the top of the oscillations, suggests analysing the data by comparing it with functions of the form

$$
y=M+A \cos (\omega t+\phi)+\kappa A \cos \left[\frac{1}{2} \omega t+\phi_{e}\right] \cos (f \omega t) .
$$

Here, $M$ represents the mean of the function, the $A \cos (\omega t+\phi)$ term represents the dominant frequency (e.g. the 20 day oscillation for neutropenia, or longer for PCML), and the last term represents an envelope at a lower frequency surrounding a higher frequency oscillation. Thus $f>1$ is the ratio of the fast to slow frequencies, and $\omega$ is the dominant frequency. A graph of this function, with $f=5.5$ and $\kappa=0.6$ is shown in Fig. 3. The choice $\phi_{e}=\phi / 2$ ensures that the maximum amplitude of the envelope oscillation occurs when the dominant cycle is at its maximum.

The qualitative similarity between the graph in Fig. 3 and the data shown in Figs. 1 and 2 is encouraging, but the similarity is best quantified through frequency analysis. Since the data are not evenly sampled, the Lomb periodogram 

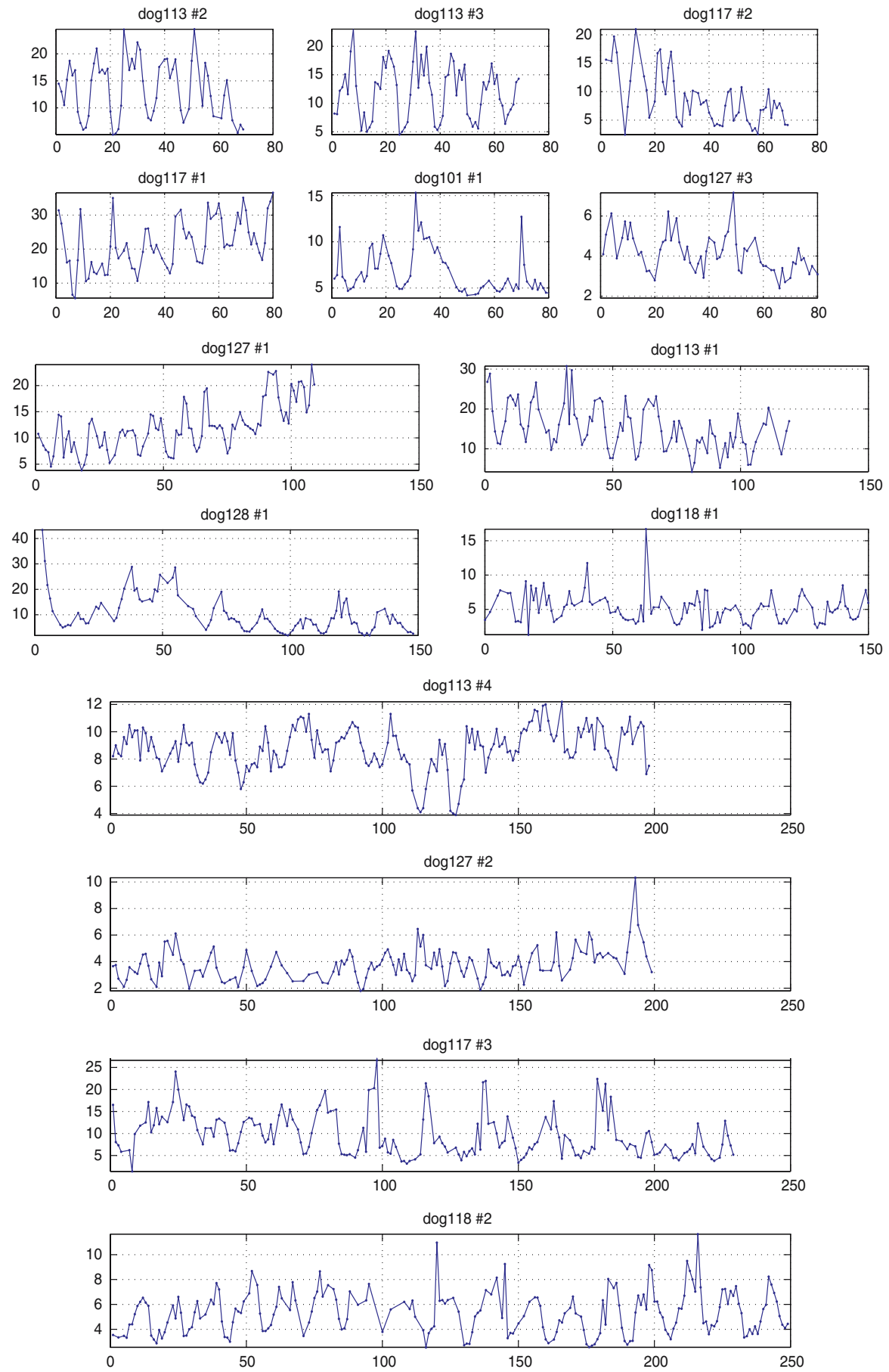

Fig. 1 Grey collie data where high-frequency oscillations are shown. Plots show neutrophil numbers in units of $\times 10^{8}$ cells kg ${ }^{-1}$, versus time in days 

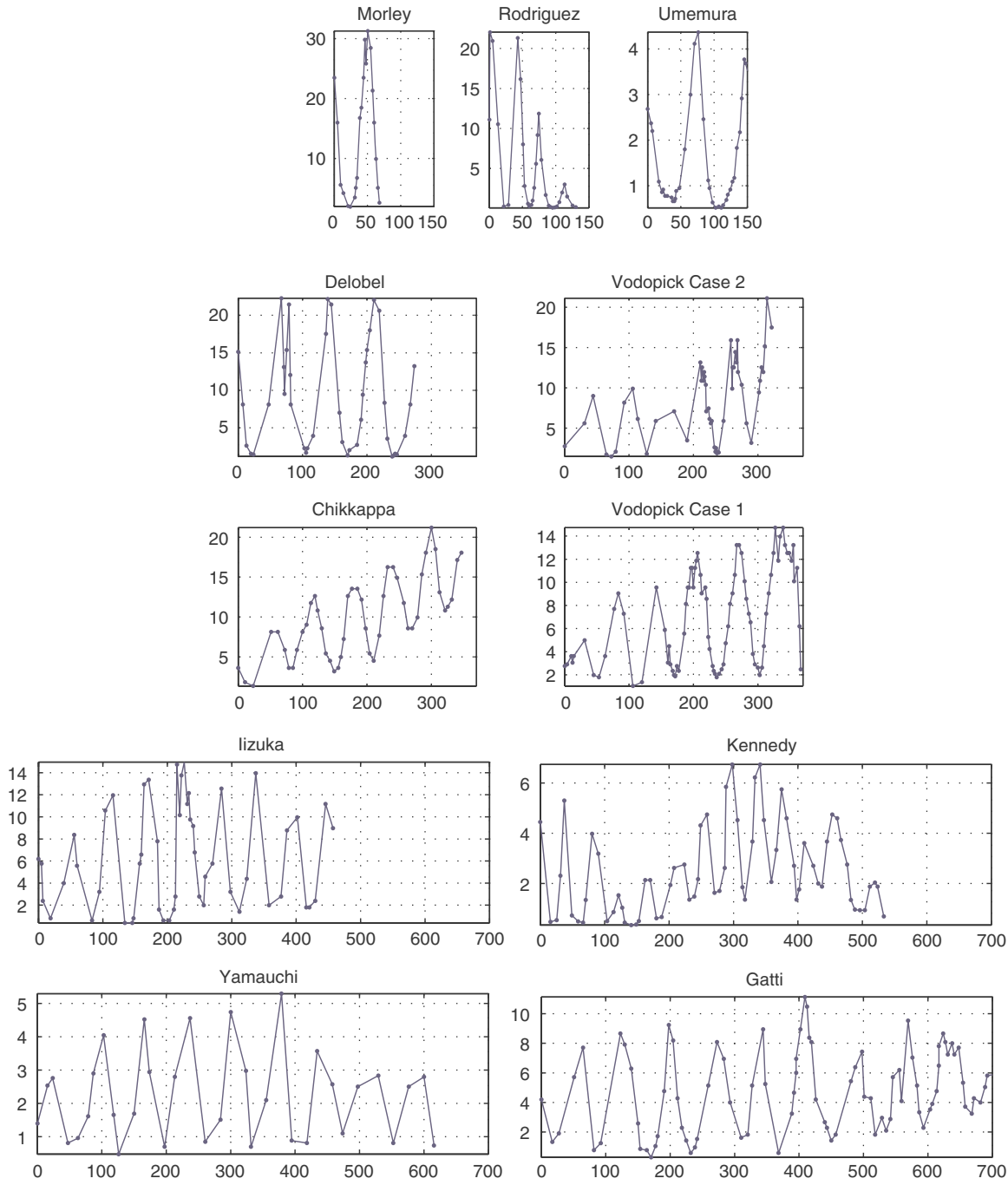

Fig. 2 Human patient data for periodic chronic myelogenous leukemia showing the high-frequency oscillations that occur when sampling is frequent, labeled by the authors of the published studies. Plots show neutrophil numbers in units of $\times 10^{9}$ cells kg-1, versus time in days. Note the high frequency oscillations in the Vodopick, Gatti and Iizuka data

may be used to find dominant frequencies and their relative strengths [18]. ${ }^{1} \mathrm{We}$ therefore perform a frequency analysis, using the Lomb periodogram, of both the data sets and of simulated data sets generated from Eq. (2.1).

\footnotetext{
${ }^{1}$ The Lomb periodogram was originally developed for astrophysical data analysis, and is analogous to a Fourier periodogram but was developed for data that are unevenly sampled in time.
} 


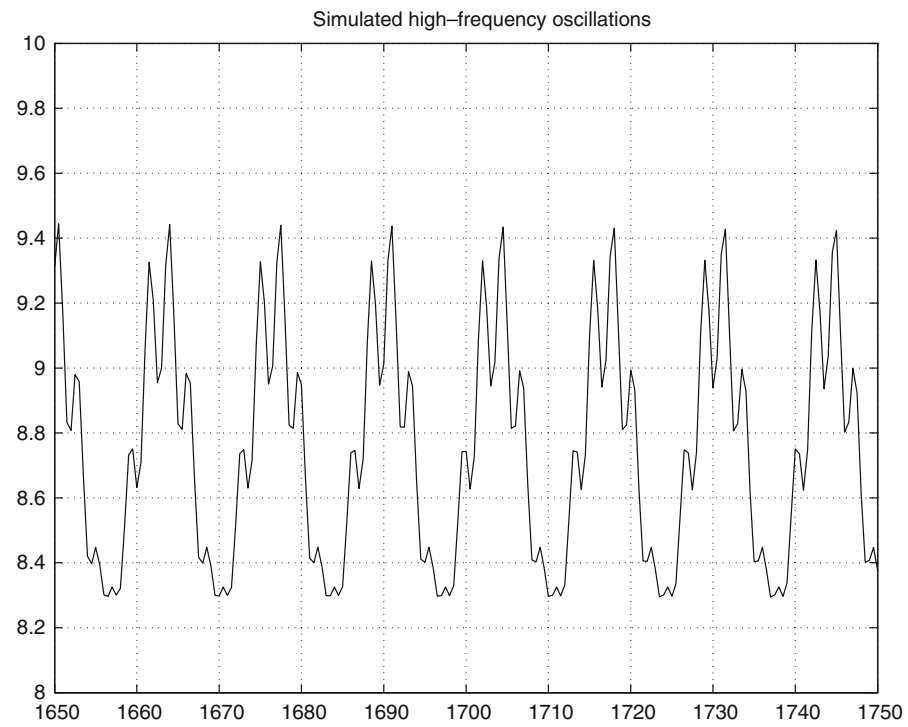

Fig. 3 The graph of the function of Eq. (2.1) with $f=5.5$ and $\kappa=0.6$

Figure 4 shows the Lomb periodograms of the data given in Fig. 1. The broken lines show the various significance levels; a level of 0.01 is taken to indicate significant periodicity. Note that a large peak at a very low frequency indicates that there is overall upward or downward drift in the data. An important feature of these plots is that in almost all cases, there is a secondary peak in the Lomb periodogram at twice the main frequency. While this has been documented in cyclical neutropenia previously [14], this was in untreated $\mathrm{CN}$ and was attributed to the characteristic 'second bump' in classic $\mathrm{CN}$ neutrophil data. In our data, this characteristic second bump is not visible, yet the Lomb periodogram frequently displays a peak at twice the dominant frequency. We also see an additional peak above that. Thus, the data are not composed simply of a superposition of the main frequency and twice the main frequency.

The relevant question is now whether surrogate data, created with the aid of Eq. (2.1), can mimic the observed Lomb periodograms, especially for the dog data shown in Fig. 1 where the high frequency oscillation occurs quite regularly.

The approximate waveform in Eq. (2.1) (or a simplified variant without the phases) can be written in terms of the frequencies

$$
\begin{aligned}
& A \cos (\omega t)+\kappa A \cos \left[\frac{1}{2} \omega t\right] \cos (f \omega t) \\
& \quad=A \cos (\omega t+\phi)+\frac{\kappa A}{2} \cos \left[-\frac{1}{2} \omega t+f \omega t\right]+\frac{\kappa A}{2} \cos \left[\frac{1}{2} \omega t+f \omega t\right]
\end{aligned}
$$

so that if the second peak is to be at twice the main peak, we find $f=2.5$ by setting $-\frac{1}{2} \omega+f \omega=2 \omega$. However, $f$ may be higher, because with the fixed (and somewhat low) sampling rate, a peak at $2 \omega$ appears in the Lomb periodogram 

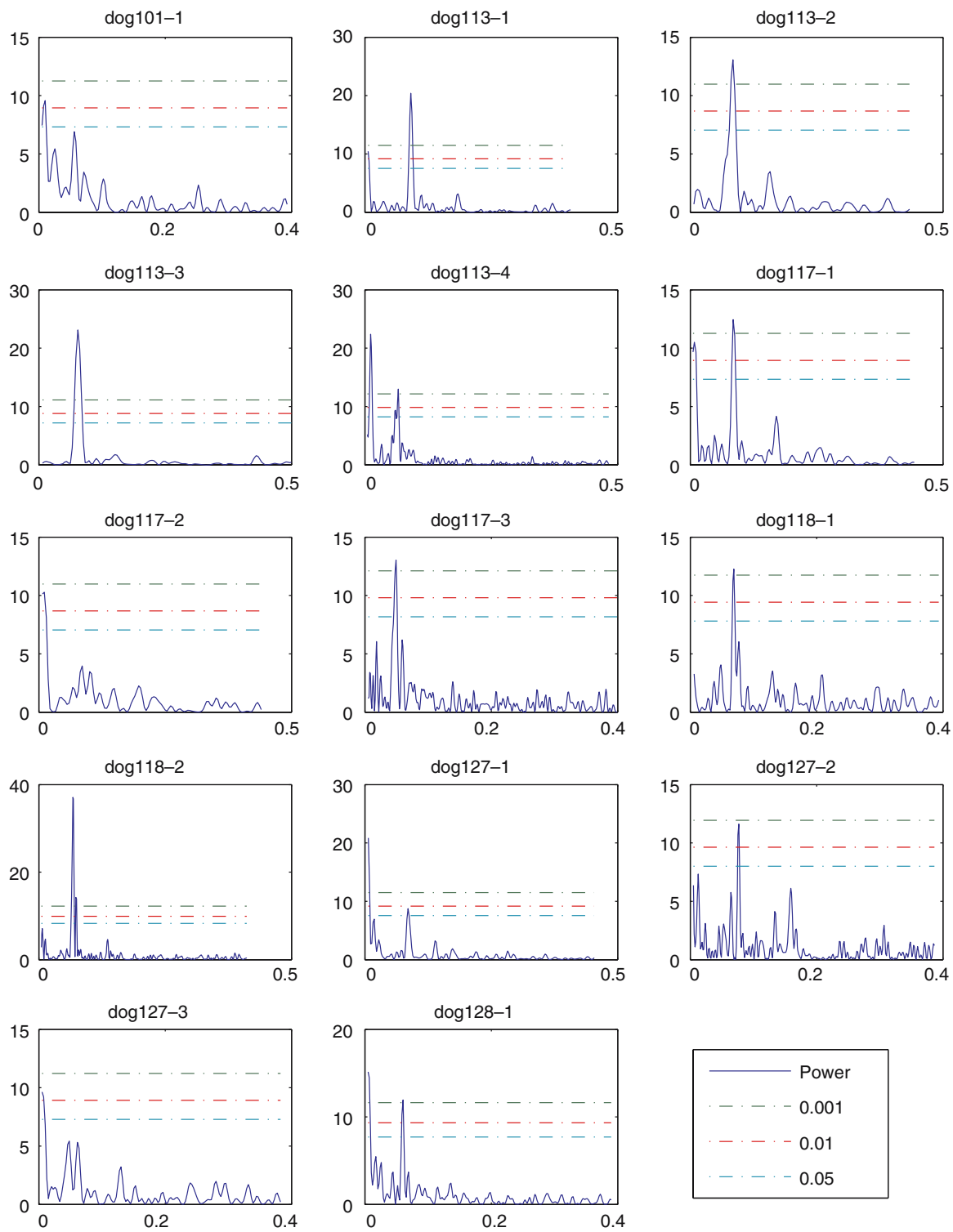

Fig. 4 The corresponding Lomb periodograms of the data shown in Fig. 1. The horizontal axis is the frequency in days ${ }^{-1}$ and the vertical axis is the power of the corresponding frequency. Dashed lines show significance levels as indicated in the legend

even when $f=5$. This is due to the fact that there is a maximum frequency that the periodogram can detect at a given sampling rate, together with the fact that a function of period $T$ is also periodic with period $n T$ for integer $n$.

We created a surrogate data set using Eq. (2.1) in the following way. First, the dominant frequency of each data set is found using the Lomb periodogram. Using that frequency as a starting value (and reasonable estimates for the mean 
and phase), a nonlinear fit procedure is carried out to match the data to a function of the form of Eq. (2.1). The fit was performed with Matlab's nlinfit function, which uses the Gauss-Newton algorithm with Levenberg-Marquardt modifications. The resulting function is then sampled at the same times that the data are sampled to create the surrogate data. The corresponding Lomb periodograms of the surrogate data, compared to the Lomb periodograms of the original data, are shown in Fig. 5. In this figure, the frequency scale $f$ is always approximately 2.5 .

From the figure, note that many of the secondary peaks are aligned in the Lomb periodograms of the surrogate and real data. However, it is natural that not all the features of the real data are matched by this relatively simple comparison; for example, from the analytically determined Fourier transform of Eq. (2.1), only three peaks will appear in the frequency spectrum of the surrogate data, where the real data have more complex frequency compositions. This can be due to time-dependent physiological parameters, random fluctuations, the presence of other harmonics in the dynamics (which in real life are more complex than those in the model), etc. However the matching of the frequency spectra of the real and the surrogate data lends credibility to the notion that the data consistently have higher frequency oscillations, occurring mainly at the top of the dominant oscillation in the neutrophils. It is this hypothesis that we will explore, from the perspective of the dynamics of the model, in the rest of this paper.

\subsection{Oscillations in human periodic chronic myelogenous leukemia}

We also have data from several patients with a periodic form of leukemia (periodic chronic myelogenous leukemia or PCML), previously analysed by Fortin and Mackey [8], showing rapid variation in leukocyte numbers during periods when the sampling rate was relatively high. Furthermore, high frequency oscillations were seen in model simulations [4] that were fitted to the PCML data using a simulated annealing algorithm.

Figure 2 shows white blood cell count data for PCML patients. Note that in the data published by Iizuka, Delobel, Gatti, and Vodopick, when sampling times are more frequent, high frequency variation is seen. (The references for the PCML data are: $[3,7,10,15,16,19,21-23,26]$.)

Figure 6 shows the Lomb periodograms of the PCML data. Here, we do not see any obvious consistent behavior in the Lomb periodograms other than the main frequency of the oscillations. However, this is almost certainly because for most of the cycles in the data, the sampling times were too sparse to pick up any higher frequency oscillation, so that these higher frequencies were not sufficiently regular to appear in the Lomb periodogram.

\section{A mathematical model of stem cell/neutrophil dynamics}

Few would dispute that the data in Figs. 1 and 2 indicate periodic behavior, with periods of order 10 days for the dogs and 50 days for the humans with 

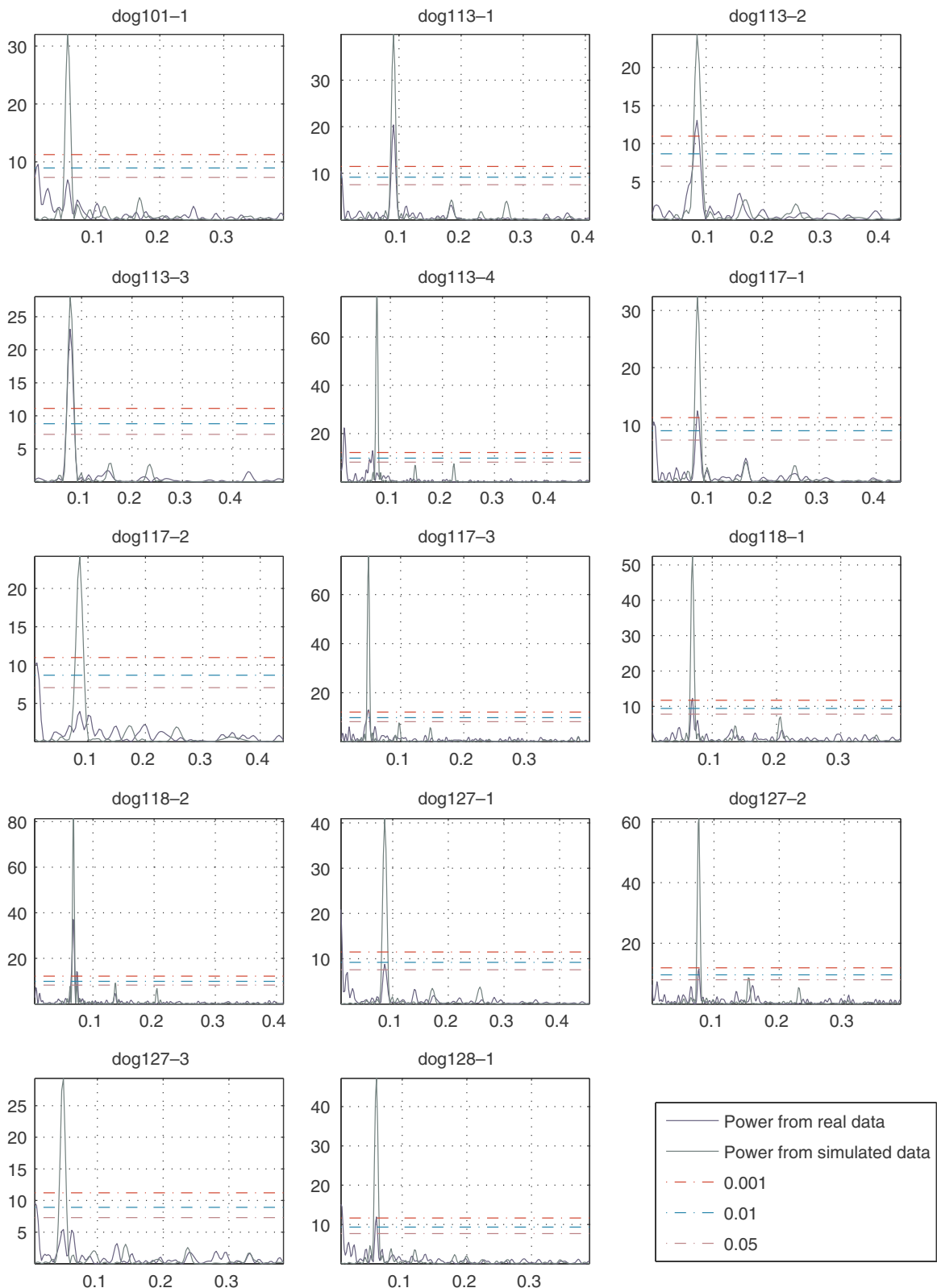

Fig. 5 Lomb periodograms of both real and surrogate data showing alignment of most secondary peaks. The horizontal axis is the frequency in days ${ }^{-1}$ and the vertical axis is the power of the corresponding frequency. Dashed lines show significance levels as indicated in the legend

PCML. It is equally clear that the data are not precisely periodic, principally through time variation of the fluctuation amplitude. The fact that despite this, the periodicity is so marked, suggests that we should pay equal attention to the 

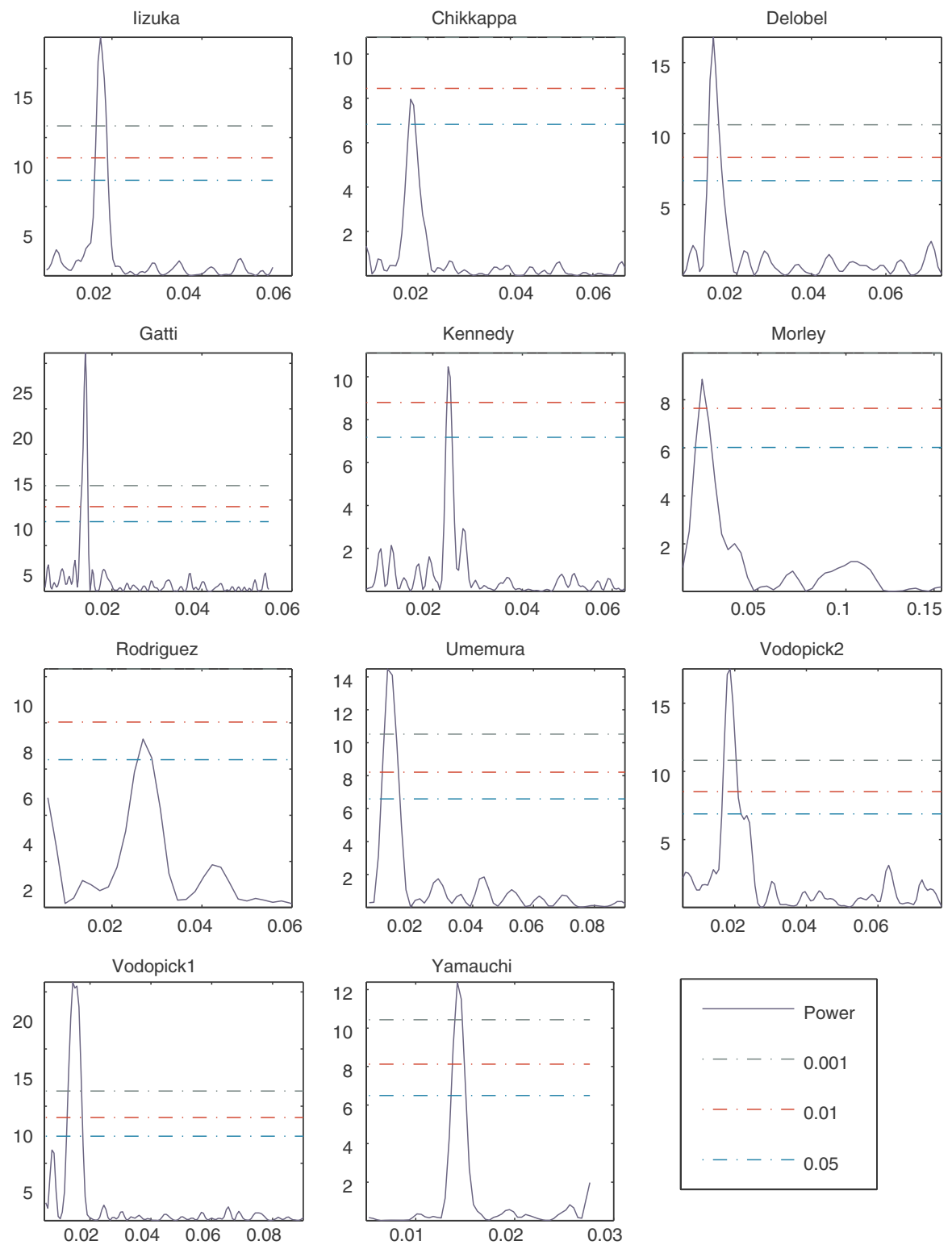

Fig. 6 Lomb periodograms of the PCML data from Fig. 2. The horizontal axis is the frequency in days ${ }^{-1}$ and the vertical axis is the power of the corresponding frequency. Dashed lines show significance levels as indicated in the legend

relatively large amplitude excursions from the periodic signal which occur on a more rapid time scale. This is seen, for example, in the data for dog 113-3 in Fig. 1. Note that where these high frequency fluctuations are seen, it is mostly when the neutrophils are at a maximum. Our object in the following sections is therefore to examine whether a mathematical model of stem cell oscillations 
which can produce long period oscillations also has the capability of predicting high frequency fluctuations, and in particular whether these can be localised on the peaks of the neutrophil oscillations.

The starting point of our study is the mathematical model presented by Colijn and Mackey [4,5] for the combined dynamics of stem cells, neutrophils, platelets and red blood cells. The dimensional version of the model is given by

$$
\begin{aligned}
& \frac{\mathrm{d} Q}{\mathrm{~d} t}=-\beta(Q) Q-\left(\kappa_{N}(N)+\kappa_{R}(R)+\kappa_{P}(P)\right) Q+2 \mathrm{e}^{-\gamma_{S} \tau_{S}} \beta\left(Q_{\tau_{S}}\right) Q_{\tau_{S}}, \\
& \frac{\mathrm{d} N}{\mathrm{~d} t}=-\gamma_{N} N+A_{N} \kappa_{N}\left(N_{\tau_{N}}\right) Q_{\tau_{N}}, \\
& \frac{\mathrm{d} P}{\mathrm{~d} t}=-\gamma_{P} P+A_{P}\left\{\kappa_{P}\left(P_{\tau_{\mathrm{PM}}}\right) Q_{\tau_{\mathrm{PM}}}-\mathrm{e}^{-\gamma_{P} \tau_{\mathrm{PS}}} \kappa_{P}\left(P_{\tau_{\mathrm{PM}}+\tau_{\mathrm{PS}}}\right) Q_{\tau_{\mathrm{PM}}+\tau_{\mathrm{PS}}}\right\}, \\
& \frac{\mathrm{d} R}{\mathrm{~d} t}=-\gamma_{R} R+A_{R}\left\{\kappa_{R}\left(R_{\tau_{\mathrm{RM}}}\right) Q_{\tau_{\mathrm{RM}}}-\mathrm{e}^{-\gamma_{R} \tau_{\mathrm{RS}}} \kappa_{R}\left(R_{\tau_{\mathrm{RM}}+\tau_{\mathrm{RS}}}\right) Q_{\tau_{\mathrm{RM}}+\tau_{\mathrm{RS}}}\right\},
\end{aligned}
$$

where we have used the notation $x_{\tau} \equiv x(t-\tau)$, and

$$
\begin{aligned}
\beta(Q) & =k_{0} \frac{\theta_{2}^{s}}{\theta_{2}^{s}+Q^{s}}, \\
\kappa_{N}(N) & =f_{0} \frac{\theta_{1}^{n}}{\theta_{1}^{n}+N^{n}}, \\
\kappa_{P}(P) & =\frac{\bar{\kappa}_{p}}{1+K_{p} P^{m}}, \\
\kappa_{R}(R) & =\frac{\bar{\kappa}_{r}}{1+K_{r} R^{r}} .
\end{aligned}
$$

Colijn and Mackey [4] develop this model in detail, and thus it will not be derived here. Essentially, the pluripotential hematopoietic stem cells $Q$ can proliferate or they can differentiate into the three peripheral cell lines. Thus, in the model, they re-enter the proliferative phase at a rate $\beta(Q)$, or differentiate at rates $\kappa_{N}(N), \kappa_{R}(R)$ and $\kappa_{P}(P)$. All of these involve negative feedbacks that take the form of Hill functions. The feedback functions have delayed arguments, due to the several days required for cells to proliferate or mature. Table 1 contains a list and description of the delays. Figure 7 shows a schematic diagram of the model.

In dimensionless form, these equations are

$$
\begin{aligned}
\dot{q} & =-q\left[b_{1} h_{1}(q)+b_{2} h_{2}(n)+b_{3} h_{3}(p)+b_{4} h_{4}(r)\right]+b_{1} \lambda_{1} q_{1} h_{1}\left(q_{1}\right), \\
\dot{n} & =-\gamma_{n} n+A_{n} b_{2} q_{\tau_{n}} h_{2}\left(n_{\tau_{n}}\right), \\
\dot{p} & =-\gamma_{p} p+A_{p} b_{3}\left\{q_{\tau_{p 1}} h_{3}\left(p_{\tau_{p 1}}\right)-\lambda_{3} q_{\tau_{p 2}} h_{3}\left(p_{\tau_{p 2}}\right)\right\}, \\
\dot{r} & =-\gamma_{r} r+A_{r} b_{4}\left\{q_{\tau_{r 1}} h_{4}\left(r_{\tau_{r 1}}\right)-\lambda_{4} q_{\tau_{r 2}} h_{4}\left(r_{\tau_{r 2}}\right)\right\} .
\end{aligned}
$$


Fig. 7 Schematic representation of the model

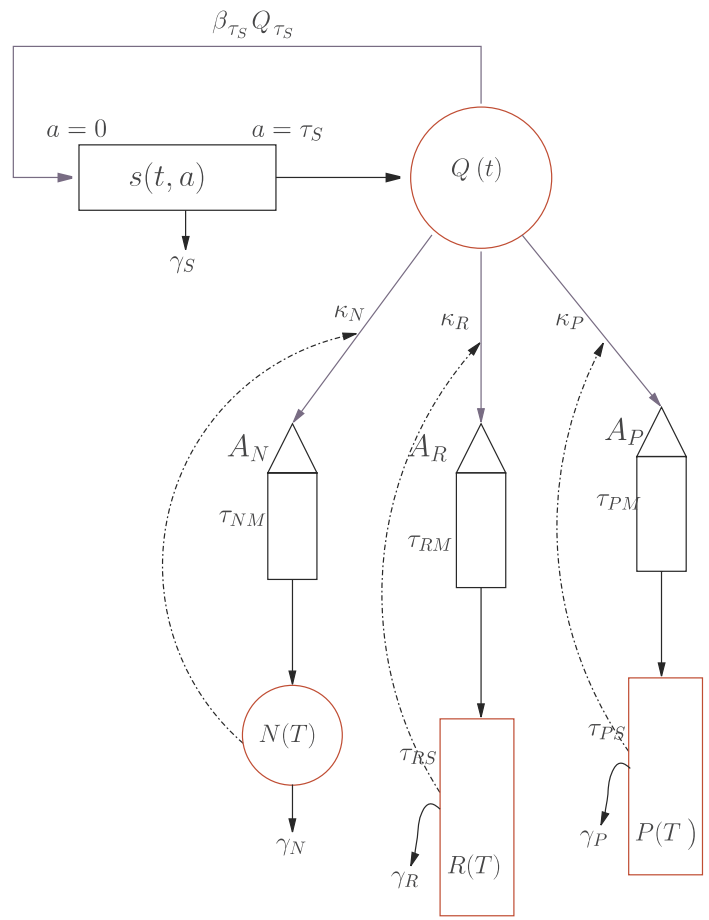

Table 1 Delays in the model

\begin{tabular}{ll}
\hline Delay & Description \\
\hline$\tau_{S}$ & Stem cell proliferation time \\
$\tau_{N}$ & Leukocyte maturation time \\
$\tau_{\mathrm{RM}}$ & Erythrocyte maturation time \\
$\tau_{\mathrm{RS}}$ & Erythrocyte aging time to senescence \\
$\tau_{\mathrm{Rsum}}$ & $\tau_{\mathrm{RM}}+\tau_{\mathrm{RS}}$ \\
$\tau_{\mathrm{PM}}$ & Platelet maturation time \\
$\tau_{\mathrm{PS}}$ & Platelet aging time to senescence \\
$\tau_{\mathrm{Psum}}$ & $\tau_{\mathrm{PM}}+\tau_{\mathrm{PS}}$ \\
\hline &
\end{tabular}

The variables are $q$, stem cell population; $n$, neutrophil population; $p$, platelet population; and $r$, red blood cell population: an overdot denotes differentiation with respect to $t$. Subscripts on these variables, such as $q_{1}$ and $q_{\tau_{n}}$ indicated delayed arguments, with the subscript indicating the delay; thus $q_{1} \equiv q(t-1)$, and so on. The functions $h_{i}$ are Hill-type controlling functions, and specifically

$$
\begin{array}{ll}
h_{1}(q)=\frac{1}{1+q^{s_{1}}}, & h_{2}(n)=\frac{1}{1+n^{s_{2}}}, \\
h_{3}(p)=\frac{1}{1+p^{s_{3}}}, & h_{4}(r)=\frac{1}{1+r^{s_{4}}} .
\end{array}
$$

Typical operating values of the parameters in normal circumstances are given in Table 2. Neutropenic oscillations are simulated (c.f. Colijn and Mackey [5]) 
Table 2 Definitions and typical values of the dimensionless parameters of the model

\begin{tabular}{lll}
\hline Symbol & Definition & Typical value \\
\hline$b_{1}$ & $\tau_{S} k_{0}$ & 22.4 \\
$b_{2}$ & $\tau_{S} f_{0}$ & 1.1 \\
$b_{3}$ & $\tau_{S} \bar{\kappa}_{P}$ & 3.3 \\
$b_{4}$ & $\tau_{S} \bar{\kappa}_{R}$ & 3.3 \\
$s_{1}$ & $s$ & 4 \\
$s_{2}$ & $n$ & 1 \\
$s_{3}$ & $m$ & 1.3 \\
$s_{4}$ & $r$ & 7 \\
$\lambda_{1}$ & $2 \mathrm{e}^{-\gamma_{S} \tau_{S}}$ & 1.6 \\
$\lambda_{3}$ & $\mathrm{e}^{-\gamma_{P} \tau_{\mathrm{PS}}}$ & 0.9 \\
$\lambda_{4}$ & $\mathrm{e}^{-\gamma_{R} \tau_{\mathrm{RS}}}$ & 0.24 \\
$\gamma_{n}$ & $\tau_{S} \gamma_{N}$ & 6.7 \\
$\gamma_{r}$ & $\tau_{S} \gamma_{R}$ & 0.003 \\
$\gamma_{p}$ & $\tau_{S} \gamma_{P}$ & 0.42 \\
$A_{n}$ & $A_{N} \theta_{2} / \theta_{1}$ & 700 \\
$A_{p}$ & $A_{P} \theta_{2} K_{P}^{1 / m}$ & 58 \\
$A_{r}$ & $A_{R} \theta_{2} K_{R}^{1 / r}$ & 1.1 \\
$\tau_{n}$ & $\tau_{N M} / \tau_{S}$ & 1.25 \\
$\tau_{p 1}$ & $\tau_{\mathrm{PM}} / \tau_{S}$ & 2.5 \\
$\tau_{p 2}$ & $\tau_{\mathrm{Psum}} / \tau_{S}$ & 5.9 \\
$\tau_{r 1}$ & $\tau_{\mathrm{RM}} / \tau_{S}$ & 2.1 \\
$\tau_{r 2}$ & $\tau_{\mathrm{Rsum}} / \tau_{S}$ & 45 \\
\hline
\end{tabular}

if $A_{n}$ is reduced (range: 50-250), $b_{1}$ is reduced (range: 6-15), and $b_{2}$ increased slightly (range: $1-3)$.

\section{Spiky oscillations}

The framework for our analysis is the result of Fowler and Mackey [9], who showed that long period oscillations (that is to say, long compared with stem cell cycling time, here scaled to be one) would occur if the net reproduction rate was small, which in the present context means $\lambda_{1}-1$ is small. Although the value $\lambda_{1}-1=0.6$ hardly appears small, we shall proceed formally as if it were. The resulting narrative will therefore have a qualitative applicability, rather than quantitative accuracy.

\subsection{Long period oscillations}

We will begin by supposing that $p$ and $r$ are essentially steady. Because $A_{p} b_{3} / \gamma_{p}$ is large, then $p \sim A_{p} b_{3} q h_{3}(p) / \gamma_{p}$, and this suggests that $h_{3}(p)$ will be small. Similar reasoning suggests that $h_{4}(r)$ will be small. So we begin by neglecting these terms in the first equation of (3.3).

Our basic ansatz for the long period relaxation oscillations is that $q$ varies slowly with $t$ during two phases of the oscillation, between which fast transitions occur (see [9]). In contrast, $n$ changes on a time scale $t \sim 1 / \gamma_{n} \sim 0.15$ which is 
small, so that we can expect $n$ to relax to a quasi-steady state $\bar{n}$ in which

$$
\bar{n} \approx \frac{A_{n} b_{2} q_{\tau_{n}} h_{2}(\bar{n})}{\gamma_{n}} .
$$

Since $\frac{A_{n} b_{2}}{\gamma_{n}}$ is large, we may take

$$
h_{2}(n) \approx n^{-s_{2}},
$$

and thus

$$
\bar{n} \approx\left(\frac{A_{n} b_{2} q_{\tau_{n}}}{\gamma_{n}}\right)^{1 /\left(1+s_{2}\right)} .
$$

Hence $q$ satisfies the approximate equation

$$
\dot{q} \approx-q\left[b_{1} h_{1}(q)+\frac{\beta}{q_{\tau_{n}}^{s_{2} /\left(1+s_{2}\right)}}\right]+b_{1} \lambda_{1} q_{1} h\left(q_{1}\right)
$$

where

$$
\beta=b_{2}^{1 /\left(1+s_{2}\right)}\left(\frac{\gamma_{n}}{A_{n}}\right)^{s_{2} /\left(1+s_{2}\right)},
$$

and is small.

Now if $q$ varies slowly, $\dot{q}$ must be small, which requires $\lambda_{1} \approx 1$ and $\beta \ll 1$. We may then carry out a Taylor series expansion of the delayed terms about the current time, and this gives us, to leading order (replacing $\lambda_{1}$ by 1 in the denominator),

$$
\dot{q} \approx \frac{\left(\lambda_{1}-1\right) G(q)-\beta q^{1 /\left(1+s_{2}\right)}}{1+G^{\prime}(q)},
$$

where we define

$$
G(q)=b_{1} q h_{1}(q)
$$

Our ansatz that $q$ varies slowly is thus justified if the expression in (4.6) is reasonably small.

If this is the case, then the relaxation oscillations can be graphically portrayed with reference to Fig. 8, which shows the graph of the function $G(q)$ with values $b_{1}=10, s_{1}=4$. When oscillations occur, they consist of slow branches $A B$ and $C D$, when $q$ varies as indicated by the arrows. At the points $B$ and $D$, $G^{\prime}(q)=-1$, and there are rapid transitions $B C$ and $D A$ which connect the two slow branches. Note that the denominator $1+G^{\prime}(q)$ of (4.6) is positive except when $q$ is between $B$ and $D$. The issue of whether oscillations occur is thus controlled by the numerator of (4.6). When $q$ is large enough, this numerator 
Fig. 8 The function $G(q)=b_{1} q h_{1}(q)$ for values $b_{1}=10, s_{1}=4$. Also shown are the unit slope transition lines $6.476-q$ and $3.093-q$
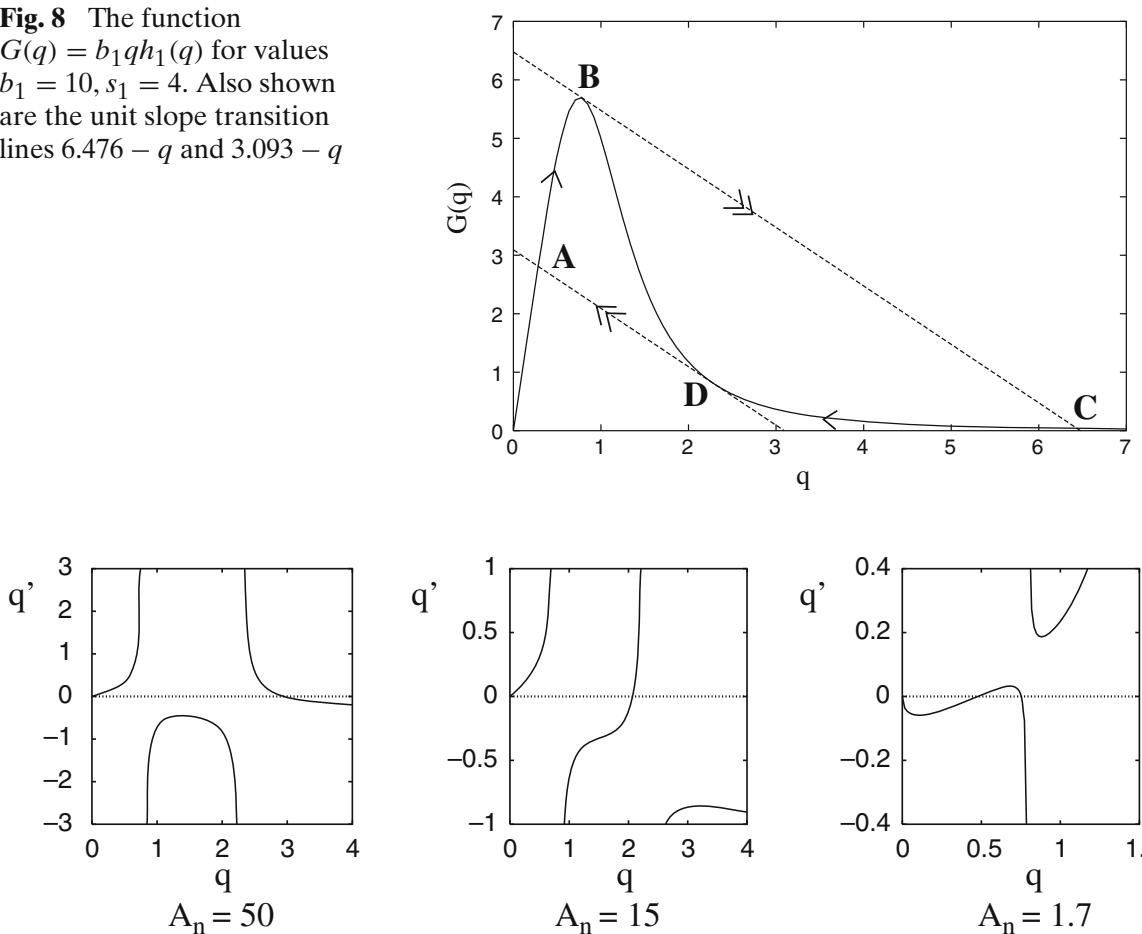
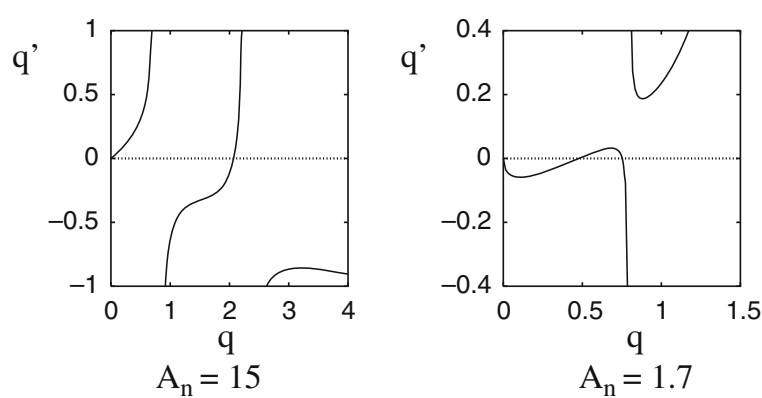

Fig. 9 The function $q^{\prime}$ given by (4.6) with the default parameters given in Table 2, excepting that $b_{1}=10$, and the values of $A_{n}$ are as shown

has a unique zero, and oscillations occur when it lies between $B$ and $D$. The reason for this is illustrated in Fig. 9, which shows the graph of $q^{\prime}$ versus $q$ for three different values of $A_{n}$ (other parameters being fixed). When $A_{n}$ is large, $\beta$ is small, and there is a large positive zero of $q^{\prime}$, which corresponds to a stable steady state. As $A_{n}$ is decreased, $\beta$ increases, and this zero moves progressively to the left, until in the central panel of Fig. 9 it lies between $B$ and $D$ of Fig. 8. It is in this case that an oscillation occurs. Referring to Figs. 8 and 9, we see that when $A_{n}=15, \dot{q}>0$ for $q<q_{B}$, and $\dot{q}<0$ for $q>q_{D}$; thus $q$ moves slowly towards $B$ and $D$ in these respective regions, and when $q$ reaches either point, there is a rapid jump along the transition lines in Fig. 8 towards the other slow region.

The conclusion of our discussion concerning long period $q$ oscillations is that as the neutrophil production rate $A_{n}$ decreases, the healthy steady state for $q$ becomes unstable, providing also that the stem cell renewal rate $\lambda$ is sufficiently small. In the following section, we revisit the dynamics of the neutrophil population $n$, bearing these observations in mind. Note that the slow variation of $q$ is formally justified, from (4.6), if $\lambda-1 \ll 1$ and $\beta \ll 1$. The latter of these is reasonable, but for $\lambda=1.6$ it is marginal. Figure 9 shows that the assumption is just about tenable. 


\subsection{Excitable transients}

We return to the equation describing the neutrophil population $n$ :

$$
\dot{n}=-\gamma_{n} n+A_{n} b_{2} q_{\tau_{n}} h_{2}\left(n_{\tau_{n}}\right) .
$$

Because $n$ varies on a rapid time scale, the discussion concerning slow oscillations of $q$ should remain valid even if $n$ varies in time, providing $\bar{n}$ is defined in that discussion by its local average

$$
\bar{n}=\frac{1}{T} \int_{t-T}^{t} n \mathrm{~d} t,
$$

where we take $T$ to be much larger than $1 / \gamma_{n}$, but much less than the time over which $q$ varies. It is evident from (4.8) that $\bar{n}$ is defined very similarly to its definition in (4.1) (the two are the same if we assume

$$
\left.\overline{h_{2}(n)}=h_{2}(\bar{n})\right) \text {, }
$$

so in fact exactly the same discussion applies. For simplicity, we will take (4.10) to be the case, so that (4.1) applies.

Now, given that $q$ varies slowly in time (except during the fast transitions - but even these are slower than the neutrophil time scale), we see that (4.8) is a first order delay-recruitment equation for $n$ with a delayed negative feedback term. It is well known that the (unique positive) steady state of such equations can be oscillatorily unstable if the delay is large enough. Denoting [as in (4.9)] the quasi-steady state as $\bar{n}$, we ascertain its stability by putting $n=\bar{n}+N$, linearising the resultant equation for $N$, and seeking solutions of the form $N \propto \exp (\sigma t)$. The result of this is that $\sigma$ is given by

$$
\sigma=\frac{\Sigma}{\tau_{n}}, \quad \Sigma=-\alpha-\Gamma \mathrm{e}^{-\Sigma}
$$

where

$$
\alpha=\gamma_{n} \tau_{n}, \quad \Gamma=A_{n} \tau_{n} b_{2}\left|h_{2}^{\prime}(\bar{n})\right| q .
$$

The transcendental equation for $\Sigma$ in (4.11) has an infinite number of roots, almost all of them in the left hand complex $\Sigma$ plane. When $\alpha$ and $\Gamma$ are positive, as here, instability first occurs when $\Gamma>\Gamma_{1}(\alpha)$, where $\Gamma_{1}(\alpha)$ is a positive, monotone increasing function of $\alpha$ given by

$$
\begin{aligned}
\Omega & =-\alpha \tan \Omega, \quad \Omega \in\left(\frac{\pi}{2}, \pi\right), \\
\Gamma_{1} & =\frac{\Omega}{\sin \Omega} ;
\end{aligned}
$$


$\Omega$ is the frequency of the incipient oscillation at the instability threshold. As $\alpha \rightarrow 0, \Omega \rightarrow \pi / 2$ and $\Gamma_{1} \rightarrow \pi / 2$, while as $\alpha \rightarrow \infty, \Omega \rightarrow \pi$ and

$$
\Gamma_{1} \approx \alpha+\frac{\pi^{2}}{2 \alpha}
$$

From Table $2, \alpha \approx 8.3$ and is large, so that (4.14) is appropriate. From (4.12) and (4.14) we thus have instability if

$$
\frac{A_{n} b_{2}\left|h_{2}^{\prime}\right| q}{\gamma_{n}} \gtrsim 1+\frac{\pi^{2}}{2 \alpha^{2}} .
$$

From (3.4), we have

$$
\left|h_{2}^{\prime}\right|=\frac{s_{2} h_{2} n^{s_{2}}}{n\left(1+n^{s_{2}}\right)},
$$

and (4.15) and (4.16), together with (4.1), then imply that (4.15) can be written as

$$
\frac{s_{2} A_{n} b_{2} q}{\gamma_{n}} \gtrsim\left(1+\frac{\pi^{2}}{2 \alpha^{2}}\right) \frac{A_{n} b_{2} q}{\gamma_{n}}\left(1+\frac{1}{n^{s_{2}}}\right) .
$$

At leading order, since $n \gg 1$ (compare (4.2)), this gives the instability criterion as

$$
s_{2} \gtrsim 1+\frac{\pi^{2}}{2 \alpha^{2}} .
$$

A more accurate expression is obtained if we retain the term in $1 / n^{s_{2}}$ in (4.17). Using (4.3) for $n$, we find that (4.17) takes the form

$$
q \gtrsim \frac{\gamma_{n}}{A_{n} b_{2}}\left[\frac{\left(1+\left(\pi^{2} / 2 \alpha^{2}\right)\right)}{s_{2}-1-\left(\pi^{2} / 2 \alpha^{2}\right)}\right]^{\left(1+s_{2}\right) / s_{2}}
$$

and bearing in mind (4.18), this can be equivalently accurately represented by

$$
q \gtrsim \frac{\gamma_{n}}{A_{n} b_{2}}\left[\frac{s_{2}}{s_{2}-1-\left(\pi^{2} / 2 \alpha^{2}\right)}\right]^{\left(1+s_{2}\right) / s_{2}} .
$$

With the choice of parameters in Table 2, we have $s_{2}=1$, so that the quasisteady state is stable - but only just. Since $s_{2}$ is close to the threshold (4.18), we sense that if $q$ is large enough, then oscillations of $n$ may occur through excitation of the quasi-steady state $\bar{n}$. This is exactly what happens at the fast transitions of $q$, and it suggests that for sufficiently large $q$, a transient oscillation in $n$ may occur. This is essentially what is seen in parts of Figs. 1 and 2, particularly in some of the dog data, e. g., dogs 101-1, 113-2, 113-3, 127-3 and 128-1. 


\subsection{Stable oscillations}

The choice $s_{2}=1$ was taken from Bernard et al. [1]. However, there is little to say that a value greater than one is inappropriate. If $s_{2} \gtrsim 1$, then a genuine Hopf bifurcation will occur for large enough $q$. In this case, we expect the jump at a fast $q$ transition to initiate oscillations, but it is less likely that such oscillations will be stable at the minimum values of $q$. Mostly, we do see oscillations at the peak amplitudes of the $q$ oscillations, for example in the data of dogs 127-1 and 118-2. Given that the long period oscillations have far from steady amplitudes, it is not surprising that the high frequency oscillations have significant fluctuations.

The parameter $s_{2}$ in the model is critical. If $s_{2}$ is greater than one, but not close to one, then the threshold $q$ for oscillations is small, and we expect permanent high frequency oscillations. For $s_{2}$ close to one, this threshold increases, and yields the intermittent high frequency oscillations which we seem to see. For $s_{2}<1$, we expect the high frequency oscillations to be absent. This appears consistent with numerical solutions of the model.

\subsection{Numerical results}

We have solved Eq. (3.3) numerically using a Runge-Kutta fourth order method. In the derivation of the model, the loss terms in the equations for $p$ and $r$ do not apply until $t>\tau_{p 2}$ and $t>\tau_{r 2}$, respectively (before these times, the loss terms depend on the initial conditions). In solving the problem numerically, it is simplest to accommodate this by taking the loss terms to be zero for these early times, which prevents the possibility of $p$ and $r$ becoming negative.

At the standard parameter values of Table 2, the full solution of (3.3) tends to a stable steady state $q^{*}, n^{*}, p^{*}, r^{*}$ as $t \rightarrow \infty$, with

$$
q^{*}=3.27, \quad n^{*}=18.9, \quad p^{*}=21.1, \quad r^{*}=7.37 .
$$

To represent the effect of a disease such as neutropenia, we choose to examine lower values of the parameters $b_{1}$ and $A_{n}$, and higher values of $b_{2}$. Experimenting with different values of these and other parameters reveals that the model (3.3) has a bewildering variety of behaviors, and many of the features in the data of Figs. 1 and 2 can be found in the numerical solutions. It is noteworthy in the solutions that neutrophil oscillations tend to be irregular for an initial transient period of some 50 days (presumably because of the long erythrocyte life time $\tau_{r 2}$ ). This is such a long period that it is reasonable to suppose that external physiological influences will cause the dynamics to remain in a transient state. Indeed, it is evident in Figs. 1 and 2 that the time series are indeed non-stationary. This will not be revealed in the eventual oscillatory solutions we obtain, since no injection of long time secular influences is included. As one particular (and not particularly optimal) example, Fig. 10 shows examples of $q$ and $n$ obtained with $A_{n}=200, b_{1}=8$ and $b_{2}=1.4$, and other parameters 

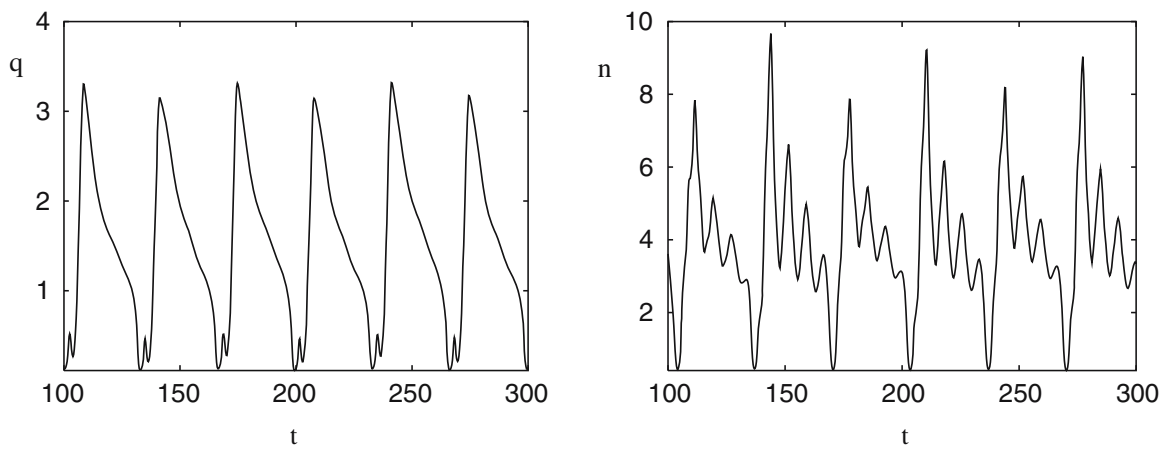

Fig. 10 Oscillatory behavior of $q$ and $n$ at parameter values $A_{n}=200, b_{1}=8, b_{2}=1.4, b_{3}=3.3$ and the others as in Table 2. The output is given in dimensional units, using a time scale $\tau_{S}=2.8$ days, a stem cell scale $\theta_{2}=0.5 \times 10^{6}$ cells kg${ }^{-1}$, and a neutrophil time scale $\theta_{1}=0.36 \times 10^{8}$ cells kg$^{-1}$. The units of time are days, those of $q 10^{6}$ cells kg-1, those of $n 10^{8}$ cells kg${ }^{-1}$. A dimensionless time step of 0.01 was used, but reasonable results are obtained with a time step of 0.1

as standard. This figure shows the behavior we have described. The neutrophil population tracks the long period oscillation of the stem cells, and a higher frequency oscillation is excited at the peak of the oscillations.

\section{Discussion}

The data we have analysed show consistent high frequency neutrophil oscillations, primarily at the peak of the neutrophil cycle. The Lomb periodogram analysis of surrogate data with these features confirms this observation. Our analysis shows that under reasonable assumptions about the parameters, the stem cell oscillations induced by a decrease in $A_{n}$ can lead to transient neutrophil oscillations when $q$ is large enough. This is precisely the phenomenon observed in the data.

One of the features of many numerical solutions which is marginally apparent in Fig. 10 is that there can be fast oscillations at the bottom of the $q$ oscillation. Although not evident in Fig. 10, these can cause an extended oscillatory minimum of $q$, and resulting high frequency neutrophil oscillations when $q$ is low. Mostly the data do not suggest such features, although there is a hint of them in the data of dog 118-1 and dog 127-3. They arise because of a property of the long period stem cell oscillation. In the transition from the slow phase $C D$ of Fig. 8, the fast transient joins the constant value of $q$ at $D$ to that at $A$. In Fowler and Mackey [9], this fast transient is described by the approximate equation

$$
\dot{q}=G\left(q_{1}\right)-G(q),
$$

where $G(q)$ is given by (4.7). The approach to the next slow phase is generally oscillatory, and whether these fast oscillations are seen depends on the sharpness of the transition, and the rapidity of the decay of the oscillation. In Fig. 10, only a few oscillations occur, but for other parameter values, there can 
be a number of these, and then the neutrophils follow suit. The parameter $s_{2}$ is closely linked to the nature of the oscillations. It is equal to 1 in our model, but if it were much larger than 1, we would expect stable oscillations in the neutrophils. If it were (as is likely) close to 1 then our analytical results predict that this would lead to the kind of intermittent high frequency oscillation seen here. Our discussion of the nature of the solutions above focusses on $q$ and $n$, and assumes that $r$ and $p$ play a passive role. Actually, $p$ satisfies an equation similar to $n$, and a similar discussion can be made for it. Like $n$, it appears to follow the slow $q$ oscillation, but has more rapid oscillatory dynamics of its own.

The red blood cells $r$ are distinguished by their very slow relaxation time (small $\gamma_{r}$ ) and large lifetime (large $\tau_{r 2}$ ). Even at 400 days in the simulation, $r$ is still relaxing transiently. The values of $p$ for the simulation of Fig. 10 are large, between 10 and 50, so that $h_{3}$ is small, but then so are $h_{2}$ and $h_{4}$. Actually, the model only makes sense if all three blood cell types are involved in the control of stem cell regulation, and this implies that $h_{2}, h_{3}$ and $h_{4}$ really should be the same size. The only real justification for considering only $n$ is that all these controllers should act in a similar way.

We are able to account for the observed intermittent high frequency oscillations with dynamical considerations of the mathematical model. It is therefore likely that there is some contribution to the high frequency oscillations from the dynamics of the system itself, and that they do not simply result from noise and the fluctuation of biological parameters.

Acknowledgments A. C. F. acknowledges with gratitude the continued support of his research by the University of Limerick. This work was supported by MITACS (Canada) and the Natural Sciences and Engineering Research Council of Canada. C. C. would like to thank the Mathematical Institute at Oxford University and A. C. F. for their hospitality during the time this research was initiated.

\section{References}

1. Bernard, S., Belair, J., Mackey, M.: Oscillations in cyclical neutropenia: New evidence based on mathematical modeling. J. Theor. Biol. 223 (2003)

2. Borge, O., Ramsfjell, V., Cui, L., Jacobsen, S.: Ability of early acting cytokines to directly promote survival and suppress apoptosis of human primitive CD34+CD38- bone marrow cells with multilineage potential at the single cell level: Key role of thrombopoietin. Blood 90, 2282-2292 (1997)

3. Chikkappa, G., G. Borner, A. H. B., Chanana, A. D., Cronkite, E. P., Ohl, S., Pavelec, M., Robertso, J. S.: Periodic oscillation of blood leukocytes, platelets, and reticulocytes in a patient with chronic myelocytic leukemia. Blood 47, 1023-1030 (1976)

4. Colijn, C., Mackey, M.: A mathematical model of hematopoiesis: I. Periodic chronic myelogenous leukemia. J. Theor. Biol. (2005a in press)

5. Colijn, C., Mackey, M.: A mathematical model of hematopoiesis: II. Cyclical neutropenia. J. Theor. Biol. (2005b in press)

6. Dale, D., Bonilla, M., Davis, M., Nakanishi, A., Hammond, W., Kurtzberg, J., Wang, W., Jakubowski, A., Winton, E., Lalezari, P., Robinson, W., Glaspy, J., Emerson, S., Gabrilove, J., Vincent, M., Boxer, L.: A randomized controlled phase iii trial of recombinant human granulocyte colony stimulating factor (filgrastim) for treatment of severe chronic neutropenia. Blood 81, 2496-2502 (1993)

7. Delobel, J., Charbord, P., Passa, P., Bernard, J.: Evolution cyclique spontanée de la leucocytose dans un cas de leucémie myéloide chronique. Nouv. Rev. franc Hémat. 13, 221-228 (1973) 
8. Fortin, P., Mackey, M.: Periodic chronic myelogenous leukemia: Spectral analysis of blood cell counts and etiological implications. Br. J. Haematol. 104, 336-245 (1999)

9. Fowler, A., Mackey, M.: Relaxation oscillations in a class of delay differential equations. SIAM J. Appl. Math. 63, 299-323 (2002)

10. Gatti, R. A., Robinson, W. A., Deinare, A. S., Nesbit, M., J. J. McCullough, M. B. . R. A. G.: Cyclic leukocytosis in chronic myelogenous leukemia. Blood 41, 771-782 (1973)

11. Haurie, C., Mackey, M. C., Dale, D. C.: Cyclical neutropenia and other periodic hematological diseases: a review of mechanisms and mathematical models. Blood 92, 2629-2640 (1998)

12. Haurie, C., Mackey, M. C., Dale, D. C.: Occurrence of periodic oscillations in the differential blood counts of congenital, idiopathic and cyclical neutropenic patients before and during treatment with G-CSF. Exper. Hematol. (1999a)

13. Haurie, C., Person, R., Dale, D. C., Mackey, M.: Haematopoietic dynamics in grey collies. Exper. Hematol. 27, 1139-1148 (1999b)

14. Haurie, C., Dale, D. C., Rudnicki, R., Mackey, M. C.: Modeling complex neutrophil dynamics in the grey collie. J. Theor. Biol. 204, 504-519 (2000)

15. Iizuka, Y., Horikoshi, A., Sekiya, S., Sawada, U., Ohshima, T., Amaki, I.: Periodic fluctuation of leukocytes, platelets and reticulocytes in a case of chronic myelocytic leukemia: The relation between leukocyte counts, cfu-c colony formation, csa and cia. Acta Haematol. Jpn. 47, 71-79 (1984)

16. Kennedy, B. J.: Cyclic leukocyte oscillations in chronic myelogenous leukemia during hydroxyurea therapy. Blood 35, 751-760 (1970)

17. Koury, M.: Programmed cell death (apoptosis) in hematopoiesis. Exp. Hematol. 20, 391-394 (1992)

18. Lomb, N. R.: Least-squares frequency analysis of unequally spaced data. Astrophys. Space Sci. 39, 447-462 (1976)

19. Morley, A. A., Baikie, A., Galton, D.: Cyclic leukocytosis as evidence for retention of normal homeostatic control in chronic granulocytic leukaemia. Lancet ?, 1320-1322 (1967)

20. Park, J.: Cytokine regulation of apoptosis in hematopoietic precursor cells. Curr. Opinion Hematol. 3, 191-196 (1996)

21. Rodriguez, A. R., Lutcher, C. L.: Marked cyclic leukocytosis leukopenia in chronic myelogenous leukemia. Am. J. Med. 60, 1041-1047 (1976)

22. Umemura, T., Hirata, J., Kaneko, S., Nishimura, J., Motomura, S., Kozuru, M., Ibayashi, H.: Periodical appearance of erythropoietin-independent erythropoiesis in chronic myelogenous leukemia with cyclic oscillation. Acta Haematol. 76, 230-234 (1986)

23. Vodopick, H., Rupp, E. M., Edwards, C. L., Goswitt, G. A., Beauchamp, J.: Spontaneous cyclic leukocytosis and thrombocytosis in chronic granulocytic leukemia. New Eng. J. Med. 286, 284-290 (1972)

24. Williams, G., Smith, C.: Molecular regulation of apoptosis: genetic controls on cell death. Cell 74, 777-779 (1993)

25. Williams, G. T., Smith, C. A., Spooncer, E., Dexter, T. M., Taylor, D. R.: Haemopoietic colony stimulating factors promote cell survival by suppressing apoptosis. Nature 353, 76-78 (1990)

26. Yamauchi, K., Ide, A.: Spontaneous remission with cyclic leukocytosis in chronic myelogenous leukemia. Acta Haematol. 88, 136-138 (1992) 